\title{
MERS Coronavirus Neutralizing Antibodies in Camels, Eastern Africa, 1983-1997
}

\section{Marcel A. Müller, ${ }^{1}$ Victor Max Corman, ${ }^{1}$ Joerg Jores, Benjamin Meyer, Mario Younan, Anne Liljander, Berend-Jan Bosch, Erik Lattwein, Mosaad Hilali, Bakri E. Musa, Set Bornstein, and Christian Drosten}

To analyze the distribution of Middle East respiratory syndrome coronavirus (MERS-CoV)-seropositive dromedary camels in eastern Africa, we tested 189 archived serum samples accumulated during the past 30 years. We identified MERS-CoV neutralizing antibodies in $81.0 \%$ of samples from the main camel-exporting countries, Sudan and Somalia, suggesting long-term virus circulation in these animals.

$\mathrm{S}$ ince 2012, a newly emerged human pathogenic coronavirus $(\mathrm{CoV})$ has caused an ongoing epidemic on the Arabian Peninsula. The designated Middle East respiratory syndrome (MERS)-CoV belongs to the Betacoronavirus genus lineage $\mathrm{C}$ and causes severe respiratory disease in humans (1). As of July 2, 2014, MERS-CoV has caused $\approx 842$ human infections, including 322 deaths (2). Dromedary camels are a putative source for MERS-CoV infection in humans. Dromedaries from countries in Africa (Egypt, Tunisia, Nigeria, Sudan, Ethiopia, and Kenya) and Arabia (United Arab Emirates, Saudi Arabia, Oman, Qatar, and Jordan) have shown high rates of MERS-CoV seropositivity in serum samples collected during the past 2 decades (3-9). In addition, MERS-CoV nucleotide sequences and virus were detected in respiratory swab samples, predominantly from juvenile dromedaries $(5,10)$. Transmission between humans and camels has been

Author affiliations: University of Bonn Medical Centre, Bonn, Germany (M.A. Müller, V.M. Corman, B. Meyer, C. Drosten); German Centre for Infection Research, Bonn (V.M. Corman); International Livestock Research Institute, Nairobi, Kenya (J. Jores, A. Liljander); Vétérinaires Sans Frontières Germany, Nairobi (M. Younan); Utrecht University, Utrecht, the Netherlands (B.-J. Bosch); EUROIMMUN AG, Lübeck, Germany (E. Lattwein); Cairo University, Giza, Egypt (M. Hilali); Ministry of Science and Communication, Khartoum, Sudan (B.E. Musa); and National Veterinary Institute, Uppsala, Sweden (S. Bornstein) described in Qatar and Saudi Arabia $(11,12)$. No autochthonous MERS-CoV infections in humans have been reported in Africa. Most dromedary camels traded in the Middle East are bred in the Greater Horn of Africa, primarily in Ethiopia, Sudan, Somalia, and Kenya (13). To further analyze the spatial and temporal distribution of MERS-CoV-seropositive camels, we tested archived camel serum samples originating in Egypt, Sudan, and Somalia, accumulated during the past 30 years, for MERS-CoV antibodies.

\section{The Study}

A serum sample from each of 189 dromedary camels was collected by trained personnel as previously described (14). Blood samples were taken by jugular vein puncture. The blood was allowed to clot and subsequently centrifuged to obtain serum, or serum was separated from the coagulated blood during slaughter. All serum samples were heat-inactivated at $56^{\circ} \mathrm{C}$ for $30 \mathrm{~min}(14)$. Serum from Somalia was collected during 1983 and 1984; samples from Sudan were collected during June and July 1984, and samples from Egypt were collected during June and July 1997. All camels from Sudan were female ( $>6$ years of age) and belonged to the Anafi breed. They were kept locally and used as a means of transport and a source of milk. The camels from Somalia were sampled at slaughterhouses in Afgoi and Mogadishu. Most camels were adults; however, detailed information about sex and age was not available. The camels from Somalia were bred predominantly for milk and meat. No background information was available for the camels from Egypt. Our study fully complied with national regulations and was approved by the ethics committee of the International Livestock Research Institute accredited by the National Council of Science and Technology in Kenya (approval no. ILRI-IREC2013-12).

We tested all serum samples for MERS-CoV antibodies at a 1:100 dilution by a recombinant MERS-CoV spike protein subunit 1-based ELISA (rELISA) as previously described $(3,12)$. To determine the assay-specific cutoff value, we tested 124 confirmed MERS-CoV antibody-negative and 106 MERS-COV antibody-positive camel serum samples from previous studies (3). For inter-assay calibration, we used the same selected positive serum samples in all applications. The optical density (OD) was measured at 450/605 $\mathrm{nm}$. We determined the OD ratio by dividing the OD of each sample by the OD of the positive serum. The cutoff was defined as the 3-fold mean OD ratio of all tested MERS-CoV antibody-negative serum samples (online Technical Appendix Figure 1, http://wwwnc.cdc.gov/EID/article/20/12/141026-Techapp1.pdf). To confirm antibody specificity and rule out possible cross-reactivity with other livestock-associated $\mathrm{CoVs}$, we conducted a highly specific MERS-CoV

${ }^{1}$ These authors contributed equally to this article. 
microneutralization test $(3,6)$. All serum samples were tested at a 1:80 dilution and at a 1:800 dilution to identify MERS$\mathrm{CoV}$ neutralizing antibodies. Serum without neutralizing activity at 1:80 was rated MERS-CoV antibody negative.

A total of $159(84.1 \%$; range among countries $80.0 \%$ $86.7 \%$ ) of 189 dromedary camels were positive for MERS$\mathrm{CoV}$ antibodies in the rELISA (Figure, Table 1). The highly specific neutralization test confirmed that $153(81.0 \%$; range $68.0 \%-86.9 \%$ ) of camels had neutralizing activity with reciprocal titers of $>80$ (Table 1). Whereas most samples (124 [65.6\%; range 56.0\%-73.8\%]) had reciprocal neutralizing titers of $80-800$, we detected high neutralizing titers of $>800$ in 29 (15.3\%; range $11.6 \%-21.7 \%$ ) samples (Table 2). Neutralizing titers correlated significantly $(\mathrm{p}<0.001$, KruskalWallis 1-way analysis of variance) with the determined OD ratios of the rELISA. The rELISA was $99.0 \%$ specific when correlated with the results of the microneutralization test (online Technical Appendix Figure 2).

MERS-CoV antibody-carrying dromedaries were present in all 3 countries in 1983, 1984, and 1997 (Figure; Tables 1,2). The high seropositivity in camels from Egypt (35 [81.4\%] of 43), a country that imports camels from Sudan and Somalia, was consistent with previous studies $(7,10)$. Strikingly, camels sampled in Somalia and Sudan $>30$ years ago were identified as MERS-CoV antibody positive with seropositivity of up to $86.7 \%$ in Sudan in 1983 .

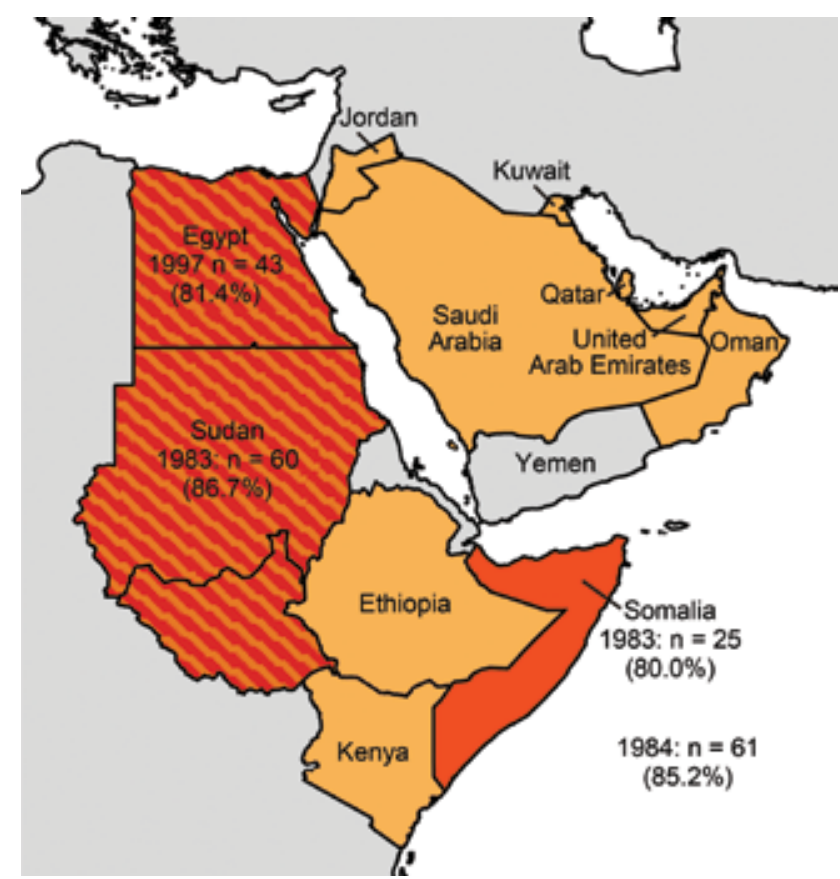

Figure. Arabian Peninsula and neighboring countries of the Greater Horn of Africa in 2014. The study sites Egypt, Sudan (separated into Sudan and South Sudan), and Somalia are in dark orange and labeled with the year the camels were sampled, the number of samples, and the percentage of samples that were reactive in the MERS-CoV ELISA. Countries with previously reported MERS-CoV seropositive dromedaries are in light orange (overlap shown in stripes).

\section{Conclusions}

Our study complements and supports the latest findings on long-term and widespread circulation of MERS-CoV or MERS-like $\mathrm{CoV}$ in dromedaries in Africa $(3,7,9,10)$. By identifying neutralizing antibodies for MERS-CoV in Somalia dromedaries, we provided data for the country lodging the world's largest camel population and from which many camels are exported to Saudi Arabia (13). The large proportion of adult animals tested in this study explains the high seropositivity $(>80 \%)$ and agrees with previous observations $(3,6,12)$. Earlier reports provided evidence for seropositive camels in Kenya and Saudi Arabia dating to the early 1990s $(3,4)$. Here we describe the presence of anti-MERS-CoV antibodies in archived serum collected $>30$ years ago, increasing the timescale for detection by an additional decade. Long-term circulation of MERS-CoV or MERS-like $\mathrm{CoV}$ in dromedaries can therefore be hypothesized. As suggested, an important factor possibly contributing to continuous virus maintenance in camels could be a high camel population density combined with nomadic husbandry, including frequent contact among camel herds in the Greater Horn of Africa (3).

MERS-CoV sequences from camels in Saudi Arabia and Qatar were closely related to sequences found in humans and did not show major genetic variability that would support long-term evolution of MERS-CoV in camels $(10,11)$. The MERS-CoV sequence from a camel in Egypt was phylogenetically most distantly related to all other known camel-associated MERS-CoVs but closely related to the early human MERS-CoV isolates (10). An urgent task would be to characterize the diversity of MERS-related $\mathrm{CoV}$ in other camels in Africa to elucidate whether the current epidemic MERS-CoV strains have evolved toward more efficient human transmissibility.

The existence of unrecognized human infections in African or Arabian countries in the past cannot be ruled out. Resource-limited African countries that have been exposed to civil unrest, such as Somalia and Sudan, are not likely to diagnose and report diagnostically challenging infections

\begin{tabular}{|c|c|c|c|}
\hline Country, date & $\begin{array}{c}\text { No. } \\
\text { samples }\end{array}$ & $\begin{array}{c}\text { No. rELISA } \\
\text { positive (\%)† }\end{array}$ & $\begin{array}{c}\text { No. mNT } \\
\text { positive }(\%) \ddagger\end{array}$ \\
\hline Egypt, 1997 Jul & 43 & $35(81.4)$ & $34(79.1)$ \\
\hline \multicolumn{4}{|l|}{ Somalia } \\
\hline 1983 Jan-Nov & 25 & $20(80.0)$ & $17(68.0)$ \\
\hline 1984 Feb-Dec & 61 & 52 (85.2) & $53(86.9)$ \\
\hline Sudan, 1983 Jun & 60 & $52(86.7)$ & $49(81.7)$ \\
\hline Total & 189 & $159(84.1)$ & $153(81.0)$ \\
\hline \multicolumn{4}{|c|}{$\begin{array}{l}\text { *MERS-CoV, Middle East respiratory syndrome coronavirus; mNT, } \\
\text { microneutralization test; rELISA, recombinant ELISA based on the MERS- } \\
\text { CoV subunit } 1 \text { spike protein. } \\
\text { †Serum was tested at a dilution of } 1: 100 \text {. } \\
\text { †mNT for MERS-CoV was done in a microtiter plate format in duplicate at } \\
\text { dilutions } 1: 80 \text { and } 1: 800 \text {. Serum with reciprocal titers }>80 \text { were considered } \\
\text { MERS-CoV antibody positive. }\end{array}$} \\
\hline
\end{tabular}


Table 2. MERS-CoV neutralizing antibody titers in dromedary camels from eastern Africa*

\begin{tabular}{lcccc}
\hline & No. & \multicolumn{3}{c}{ mNT titer, no. (\%) samples $\dagger$} \\
\cline { 3 - 5 } Country, date & samples & $<80$ & $80-800$ & $>800$ \\
\hline Egypt, 1997 Jul & 43 & $9(20.9)$ & $29(67.4)$ & $5(11.6)$ \\
\hline Somalia & & & & \\
$\quad$ 1983 Jan-Nov & 25 & $8(32.0)$ & $14(56.0)$ & $3(12.0)$ \\
1984 Feb-Dec & 61 & $8(13.1)$ & $45(73.8)$ & $8(13.1)$ \\
\hline Sudan, 1983 Jun & 60 & $11(18.3)$ & $36(60.0)$ & $13(21.7)$ \\
\hline Total & 189 & $36(19.0)$ & $124(65.6)$ & $29(15.3)$ \\
\hline *MERS-CoV, Middle East respiratory syndrome coronavirus; mNT, & \\
microneutralization test. \\
tmNT for MERS-CoV was done in a microtiter plate format in duplicate at \\
dilutions 1:80 and 1:800. Serum with reciprocal titers >80 were considered \\
MERS-CoV antibody positive.
\end{tabular}

resembling other diseases. The lack of MERS-CoV antibodies in a small cohort serosurvey in Saudi Arabia did not suggest the long-term circulation of MERS-CoV in humans on the Arabian Peninsula (15). Large serosurveys in countries where camels are bred and traded, especially in eastern Africa, are needed to explore the general MERS-CoV seroprevalence in camels and humans, particularly humans who have close contact with camels. Such serosurveys could provide the data needed to ascertain whether MERS-CoV has been introduced into, but unrecognized in, the human population on the African continent.

\section{Acknowledgments}

We thank Stephan Kallies, Monika Eschbach-Bludau, Sebastian Brünink, Tobias Bleicker, and Andrea Sieberg for excellent technical assistance. We are grateful to Tasnim Suliman for critical reading of the manuscript. We thank Fatuma M. Jama and all camel owners and herdsmen for their support during field work.

The work was funded by the European Commission (FP7EMPERIE no. 223498, FP7-ANTIGONE no. 278976) and the German Research Foundation (DFG grant DR772/3-1 to C.D.). This work was supported by the Consultative Group for International Agricultural Research (CGIAR) program on Agriculture for Nutrition and Health. The Centrum of International Migration (CIM) supported A.L. The funders had no role in study design, data collection and analysis, decision to publish, or preparation of the manuscript.

Dr Müller is staff scientist at the Institute of Virology, University of Bonn Medical Centre. His current research is dedicated to developing serologic detection methods for emerging viruses and in-depth molecular studies focusing on virus-host interactions.

\section{References}

1. Zaki AM, van Boheemen $\mathrm{S}$, Bestebroer TM, Osterhaus $\mathrm{AD}$, Fouchier RA. Isolation of a novel coronavirus from a man with pneumonia in Saudi Arabia. N Engl J Med. 2012;367:1814-20. http://dx.doi.org/10.1056/NEJMoa1211721
2. European Centre for Disease Prevention and Control. Epidemiological update: Middle East respiratory syndrome coronavirus (MERS-CoV). 02 July 2014 [cited 2014 Jul 3]. http://www. ecdc.europa.eu/en/press/news/_layouts/forms/News_DispForm. aspx?List=8db7286c-fe2d-476c-9133-18ff4cb1b568\&ID $=1029$

3. Corman VM, Jores J, Meyer B, Younan M, Liljander A, Said MY, et al. Antibodies against MERS coronavirus in dromedary camels, Kenya, 1992-2013. Emerg Infect Dis [Internet]. 2014 Aug [cited 2014 Jul 21]. http://dx.doi.org/10.3201/eid2008.140596

4. Hemida M, Perera R, Al Jassim R, Kayali G, Siu L, Wang P, et al. Seroepidemiology of Middle East respiratory syndrome (MERS) coronavirus in Saudi Arabia (1993) and Australia (2014) and characterisation of assay specificity. Euro Surveill. 2014;19:20828. http://dx.doi.org/10.2807/1560-7917.ES2014.19.23.20828

5. Hemida MG, Chu DK, Poon LL, Perera RA, Alhammadi MA, $\mathrm{Ng} \mathrm{HY}$, et al. MERS coronavirus in dromedary camel herd, Saudi Arabia. Emerg Infect Dis. 2014;20.

6. Meyer B, Muller MA, Corman VM, Reusken CB, Ritz D, Godecke GJ, et al. Antibodies against MERS coronavirus in dromedaries, United Arab Emirates, 2003 and 2013. Emerg Infect Dis. 2014;20:552-9. http://dx.doi.org/10.3201/eid2004.131746

7. Perera RA1, Wang P, Gomaa MR, El-Shesheny R, Kandeil A, Bagato $\mathrm{O}$, et al. Seroepidemiology for MERS coronavirus using microneutralisation and pseudoparticle virus neutralisation assays reveal a high prevalence of antibody in dromedary camels in Egypt, June 2013. Euro Surveill. 2013;18:20574. http://dx.doi. org/10.2807/1560-7917.ES2013.18.36.20574

8. Reusken CB, Haagmans BL, Muller MA, Gutierrez C, Godeke GJ, Meyer B, et al. Middle East respiratory syndrome coronavirus neutralising serum antibodies in dromedary camels: a comparative serological study. Lancet Infect Dis. 2013;13:859-66. http://dx.doi. org/10.1016/S1473-3099(13)70164-6

9. Reusken CBEM, Messadi L, Feyisa A, Ularamu H, Godeke GJ, Danmarwa A, et al. Geographic distribution of MERS coronavirus among dromedary camels, Africa. Emerg Infect Dis [Internet]. 2014 Dec [cited 2014 Jul 21]. http://dx.doi.org/10.3201/eid2008.140590

10. Chu DK, Poon LL, Gomaa MM, Shehata MM, Perera RA, Abu Zeid D, et al. MERS coronaviruses in dromedary camels, Egypt. Emerg Infect Dis. 2014;20:1049-53. http://dx.doi.org/10.3201/eid2006.140299

11. Haagmans BL, Al Dhahiry SH, Reusken CB, Raj VS, Galiano M, Myers R, et al. Middle East respiratory syndrome coronavirus in dromedary camels: an outbreak investigation. Lancet Infect Dis. 2014;14:140-5. http://dx.doi.org/10.1016/S1473-3099(13)70690-X

12. Memish ZA, Cotten M, Meyer B, Watson SJ, Alsahafi AJ, Al Rabeeah AA, et al. Human infection with MERS coronavirus after exposure to infected camels, Saudi Arabia, 2013. Emerg Infect Dis. 2014;20:1012-5. http://dx.doi.org/10.3201/eid2006.140402

13. Food and Agriculture Organisiation of the United Nations. FAOSTAT[cited2014Jul21].http://faostat3.fao.org/faostat-gateway/ go/to/home/E

14. Bornstein S, Musa BE. Prevalence of antibodies to some viral pathogens, Brucella abortus and Toxoplasma gondii in serum from camels (Camelus dromedarius) in Sudan. Zentralbl Veterinarmed B. 1987;34:364-70.

15. Aburizaiza AS, Mattes FM, Azhar EI, Hassan AM, Memish ZA, Muth D, et al. Investigation of anti-Middle East respiratory syndrome antibodies in blood donors and slaughterhouse workers in Jeddah and Makkah, Saudi Arabia, fall 2012. J Infect Dis. 2014;209:243-6. http://dx.doi.org/10.1093/infdis/jit589

Address for correspondence: Marcel A. Müller, Institute of Virology, University of Bonn Medical Centre, Sigmund-Freud-Str. 25, 53105 Bonn, Germany; email: muller@virology-bonn.de 